\title{
PERISACCADIC MISLOCALIZATION WITHOUT SACCADIC EYE MOVEMENTS
}

\author{
F. OSTENDORF, ${ }^{a *}$ C. FISCHER, ${ }^{a}$ B. GAYMARD ${ }^{b}$ \\ AND C. J. PLONER ${ }^{a}$ \\ ${ }^{a}$ Klinik für Neurologie, Charité, Schumannstr. 20/21, D-10117 Berlin, \\ Germany \\ 'INSERM U289, Hôpital de la Salpêtrière, 47 bd de l'Hôpital, 75651 \\ Paris Cedex 13, France
}

\begin{abstract}
Despite frequent saccadic gaze shifts we perceive the surrounding visual world as stable. It has been proposed that the brain uses extraretinal eye position signals to cancel out saccade-induced retinal image motion. Nevertheless, stimuli flashed briefly around the onset of a saccade are grossly mislocalized, resulting in a shift and, under certain conditions, an additional compression of visual space. Perisaccadic mislocalization has been related to a spatio-temporal misalignment of an extraretinal eye position signal with the corresponding saccade. Here, we investigated perceptual mislocalization of human observers both in saccade and fixation conditions. In the latter conditions, the retinal stimulation during saccadic eye movements was simulated by a fast saccade-like shift of the stimulus display. We show that the spatio-temporal pattern of both the shift and compression components of perceptual mislocalization can be surprisingly similar before real and simulated saccades. Our findings suggest that the full pattern of perisaccadic mislocalization can also occur in conditions which are unlikely to involve changes of an extraretinal eye position signal. Instead, we suggest that, under the conditions of our experiments, the arising difficulty to establish a stable percept of a briefly flashed stimulus within a given visual reference frame yields mislocalizations before fast retinal image motion. The availability of visual references appears to exert a major influence on the relative contributions of shift and compression components to mislocalization across the visual field. (C) 2005 Published by Elsevier Ltd on behalf of IBRO.
\end{abstract}

Key words: eye movements, human, extraretinal, sensorimotor integration, spatial representation.

Different theories have been proposed to explain why neither motion nor displacement of the visual scene are perceived during eye movements. Since Hermann von Helmholtz (1866), these theories predominantly suggest that the brain uses an extraretinal signal to compensate for retinal image shifts caused by saccadic eye movements. The visual system may use an efference copy (von Holst and Mittelstaedt, 1950) or corollary discharge (Sperry, 1950) of the saccadic command to cancel out the retinal translation. However, under conditions of normal illumination, space constancy could also be accomplished independently from

${ }^{*}$ Corresponding author. Tel: +49-30-450-560216; fax: +49-30-450560932.

E-mail address: florian.ostendorf@charite.de (F. Ostendorf).

0306-4522/06 $\$ 30.00+0.00$ ๑ 2005 Published by Elsevier Ltd on behalf of IBRO doi:10.1016/j.neuroscience.2005.09.032 extraretinal eye position signals by simply comparing the relative positional information available in the pre- and postsaccadic visual scene (McConkie and Currie, 1996; Deubel et al., 1996). In this context, localization of perisaccadically flashed stimuli has received intense scientific interest, since it may enable us to investigate how space constancy is achieved by the brain. A large body of evidence indicates that stimuli flashed in the dark shortly before a saccade are systematically mislocalized in saccade direction (e.g. Matin and Pearce, 1965; Honda, 1989; Cai et al., 1997). Such a uniform shift of perceived visual direction is commonly related to a presaccadic remapping of visual space, driven by a damped extraretinal eye position signal (Ross et al., 2001; Schlag and Schlag-Rey, 2002). On the other hand, a possible contribution of visual factors to presaccadic mislocalization has repeatedly been presumed (for a review, see Schlag and Schlag-Rey, 2002): Visual afferent delays and visible persistence of a briefly flashed stimulus could theoretically account for a presaccadic shift of apparent positions in saccade direction (Pola, 2004). Indeed, simulating the retinal effects of saccades by fast motion of the retinal image yields a shift of apparent positions with comparable dynamics than observed presaccadically (MacKay, 1970; O'Regan, 1984; Sperling, 1990).

Support for an additional contribution of extraretinal signals to presaccadic mislocalization comes from studies performed under conditions of normal illumination. When visual references are available, the pattern of presaccadic mislocalization changes into an additional localization bias toward the saccade target: Stimuli presented beyond the saccade target are mislocalized toward the saccade target, i.e. against saccade direction (Honda, 1993; Ross et al., 1997; Morrone et al., 1997; Lappe et al., 2000). Such a nonuniform pattern of mislocalization across the visual field has been described as an additional compression of visual space centered on the saccade target which could not be reproduced in experiments with simulated saccades (Honda, 1995; Morrone et al., 1997). The lack of space compression before simulated saccades has been taken as evidence for an additional, extraretinal signal interacting with the retinal stimulation before real saccades (Honda, 1995; Morrone et al., 1997; Ross et al., 2001; Schlag and Schlag-Rey, 2002). Alternatively, differences in retinal stimulation may account for different patterns of mislocalization before real and simulated saccades. A recent study showed the presaccadic space compression to depend on the presence of visual references (Lappe et al., 2000). Differences in the availability of visual references around image displacement may, at least partially, account for a 
lack of space compression before simulated saccades (Honda, 1995).

In the present study we investigated perceptual mislocalization shortly before saccades and saccade-like motion of the retinal image. We aimed to mimic as closely as possible the retinal stimulation around a saccadic eye movement by fast apparent motion of the visual scene. In contrast to previous studies we found highly similar spatiotemporal dynamics of mislocalization around the onset of real and simulated saccades. From our findings it may thus be inferred that visual factors play a major role in the origin of perisaccadic mislocalization.

\section{EXPERIMENTAL PROCEDURES}

\section{Subjects}

Four subjects (three male, one female, 23-27 years), served as observers in this study. They were naïve as to the purpose of the experiments and were paid for participation. Informed consent was obtained from all subjects before participation in the study which was approved by the local ethics committee and conducted in conformity with the declaration of Helsinki.

\section{Setup and protocol}

Movements of the right eye were sampled at $200 \mathrm{~Hz}$ by horizontal infrared-oculography (AMTech Eyetracker, Weinheim, Germany). Calibration was performed regularly throughout the recording session. Subjects sat in front of a 22 inch-monitor $\left(53 \times 41^{\circ}\right.$ of visual angle at $40 \mathrm{~cm}$ distance) with their head fixed to the recording system by means of a chinrest and a bitebar. Experiments were run in an otherwise darkened room. Observers fixated a point $\left(0.5^{\circ}\right)$ at screen center and made a leftward saccade to a peripheral target point which appeared at $-10^{\circ}$ and remained until the end of trial (Fig. 1A). Stimuli were black (luminance $<0.1 \mathrm{Cd} \mathrm{m}^{-2}$ ), seen against a homogenous gray background area $\left(43^{\circ} \times 41^{\circ}\right.$, luminance $1.2 \mathrm{Cd} \mathrm{m}^{-2}$ ), extending from the left screen border to $16.5^{\circ}$ right of central fixation. Screen edges were masked by black tape and not visible to the subjects. In the simulated saccade experiment, subjects maintained fixation on the fixation point at screen center when the peripheral target appeared. Two hundred milliseconds after onset of the peripheral target, the gray background area together with initial fixation and peripheral target was shifted $10^{\circ}$ rightwards (Fig. 1B). Thus after shift completion, the peripheral target took over the position at screen center, providing a new fixation point. The shift was accomplished by fast apparent motion within five video frames at a frame rate of $110 \mathrm{~Hz}$. Duration of image motion thus added up to $45 \mathrm{~ms}$. Lateral offset between
A

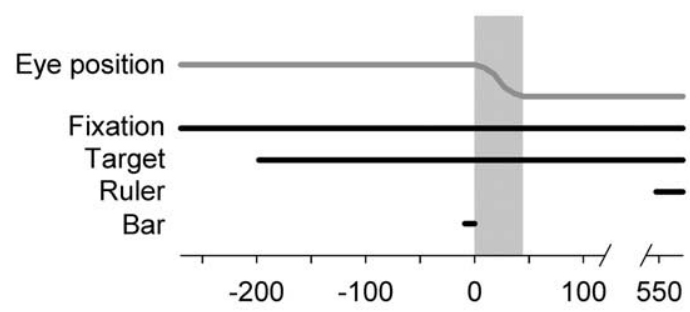

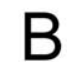

Simulated saccade

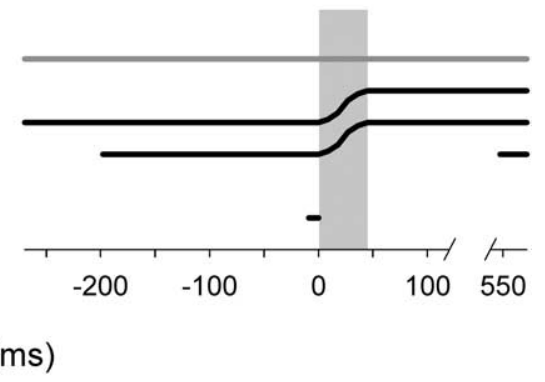

D

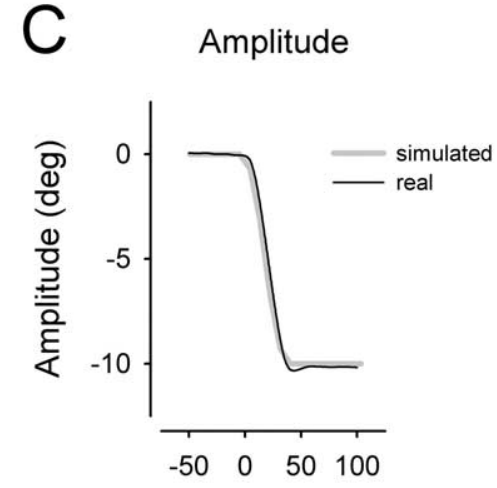

Time (msec)

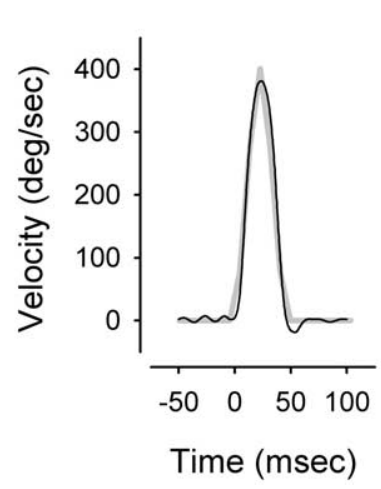

Fig. 1. Top. Schematic of the task. In the saccade task (A), observers made $10^{\circ}$ leftwards saccades from a fixation stimulus at screen center to a target stimulus appearing at $-10^{\circ}$. In the simulated saccade task (B), $200 \mathrm{~ms}$ after appearance of the target stimulus the whole visible screen area was displaced $10^{\circ}$ rightwards at saccadic speed, while observers maintained central fixation. In both tasks, at random intervals after target onset, a vertical bar was briefly flashed for $9 \mathrm{~ms}$ at either $5,-5$ or $-15^{\circ}$ eccentricity; $500 \mathrm{~ms}$ after motion offset a ruler was presented together with a mouse pointer. Bottom. Amplitude (C) and velocity profile (D) of the apparent motion used for simulating the retinal consequences of a saccade in our experiments (gray lines). The displacement was accomplished within five video frames at a frame rate of $110 \mathrm{~Hz}$. Duration of image motion thus added up to $45 \mathrm{~ms}$. Superposed is a sample saccade of one subject tested in the first experiment (black lines). 
subsequent frame positions followed a gaussian distribution, simulating the velocity profile of a saccadic eye movement (Fig. 1C, D). In both experiments, at a random interval after peripheral target onset, a vertical bar $\left(0.9 \times 41^{\circ}\right.$ of visual angle, luminance $<0.1 \mathrm{Cd} \mathrm{m}^{-2}$ ) was flashed for one frame $(9 \mathrm{~ms})$ either at $5,-5$ or $-15^{\circ}$ eccentricity. For simulated saccades, these eccentricities were defined relative to the current position of the moving initial fixation point. A reference ruler appeared together with a mouse pointer $750 \mathrm{~ms}$ after peripheral target onset. The ruler extended over the whole gray background area and had small vertical tick lines at $1^{\circ}$ intervals. Subjects moved the mouse pointer to the apparent position of the flashed bar and pressed a button. At least 222 trials were collected per subject and experiment. Further experiments with simulated saccades are described in the results section and figure legend of Fig. 4, respectively.

\section{Data analysis}

Data were analyzed offline. Saccade onset was defined by using a velocity criterion (threshold $35 \% \mathrm{~s}$ ). In the simulated saccade experiments, trials in which eye movements occurred before presentation of the reference ruler were excluded from analysis (mean: $1.27 \%$ ). It should be mentioned that by this analysis we cannot firmly exclude the occurrence of very small saccades (i.e. microsaccades) in the simulated saccade condition. Although the literature on the perceptual consequences of fixational eye movements, particularly microsaccades, is quite controversial (Martinez-Conde et al., 2004), small saccades could in principle influence our results (Reppas et al., 2002). However, we believe that the occurrence of microsaccades is unlikely to account for the results in our simulated saccade condition since the observed spatio-temporal pattern of mislocalization would necessitate a very restricted occurrence of microsaccades, time-locked to motion onset. Furthermore, a continuous visual fixation target was available around image motion and the retinal image shift was clearly shorter than previously reported microsaccadic reaction times (Horwitz and Albright, 2003).

Presentation time of the flashed bar was determined with respect to saccade onset in the saccade experiment and with respect to the start of apparent screen motion in the simulated saccade experiments. For simulated saccades, apparent bar position was determined with respect to the post-movement position of the initial fixation point.

\section{RESULTS}

\section{Mislocalization before real and simulated saccades}

Fig. 2 (left column) shows individual responses. A systematic mislocalization is observed for bars flashed around saccade onset. Consistent with previous reports (Honda, 1993, 1995; Ross et al., 1997; Morrone et al., 1997; Lappe et al., 2000), mislocalization starts approximately $50 \mathrm{~ms}$ before saccade onset, peaks at saccade onset and is nonuniform over the visual field with a large shift in saccade direction for bars presented at $5^{\circ}$ right of initial fixation and a smaller shift at $-5^{\circ}$ left of initial fixation. By contrast, at position $-15^{\circ}$ a systematic mislocalization against saccade direction evolved during saccade execution. Compared with other studies of perisaccadic mislocalization, no systematic shift toward the saccade target evolved before saccade onset for bars flashed at position $-15^{\circ}$ (Honda, 1993, 1995; Ross et al., 1997; Morrone et al., 1997; Lappe et al., 2000). However, the presaccadic shift in saccade direction is clearly nonuniform and can be described as a shift in saccade direction with an additional compression of visual space, centered around the saccade target.

In a second experiment, subjects were told to maintain fixation while the retinal image was shifted $10^{\circ}$ rightwards at saccadic speed. Fig. 2 (right column) shows the respective responses of the subjects tested in the real saccade condition described above. Here, similar dynamics of mislocalization were observed, although the shift component seems to be more prominent for simulated saccades (e.g. particularly for bars presented at $-5^{\circ}$ left of initial position, subjects SL and HP). To compare mislocalization for real and simulated saccades directly, we quantified it by means of two index measures (Lappe et al., 2000): The shift index reflects the systematic shift against direction of retinal image motion and is defined as the mean of the three apparent bar positions for a given point of time. The compression index reflects the strength of space compression toward the saccade target and is defined as the mean absolute separation between apparent bar position and saccade target $\left(-10^{\circ}\right)$, averaged over the three bar positions. Both index values are normalized with respect to their average values $100 \mathrm{~ms}$ before and after image motion. Results were comparable between subjects (Fig. 3A, C), so we averaged individual index curves. Fig. 3B, D shows the highly similar course of both index measures for real and simulated saccades. In particular, the dynamics of space compression were almost indistinguishable between real and simulated saccades while the maximal shift was larger for simulated saccades. The time-course and peak values of both measures are in good agreement with previous reports of perisaccadic mislocalization (Lappe et al., 2000).

To study the spatial distribution of mislocalization before simulated saccades in greater detail, we presented bars at multiple positions across the visual field (Morrone et al., 1997). We focused on the time of maximal mislocalization, i.e. on motion onset (Fig. 4A). Results shown in Fig. $5 \mathrm{~A}$ are consistent with the pattern of mislocalization observed at real and simulated saccade onset with the three bar positions in the experiments reported above. Mislocalization against image motion is greatest right of the initial fixation point, continuously levels off toward the peripheral target position and is essentially abolished beyond the peripheral target, implying a compression of visual space.

\section{Importance of visual references}

Our results indicate that the spatio-temporal dynamics of perisaccadic mislocalization can, at least to a certain degree, be reproduced by fast apparent motion of the retinal image. This stands in contrast to previous studies where clearly different patterns of mislocalization before real and simulated saccades were observed (Honda, 1995; Morrone et al., 1997). So far, no compression of space was found for simulated saccades.

A recent study demonstrated that the presaccadic compression of space depends on the perisaccadic availability of visual references (Lappe et al., 2000). We hypothesized that different patterns of mislocalization for real and 
Real saccades
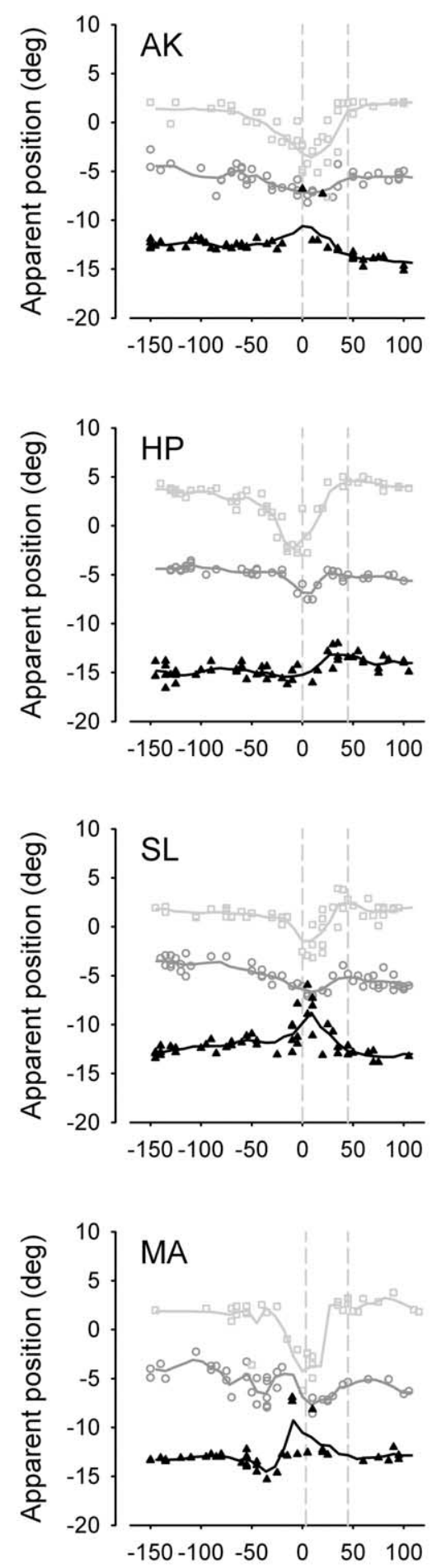

Time from saccade onset (ms)
Simulated saccades
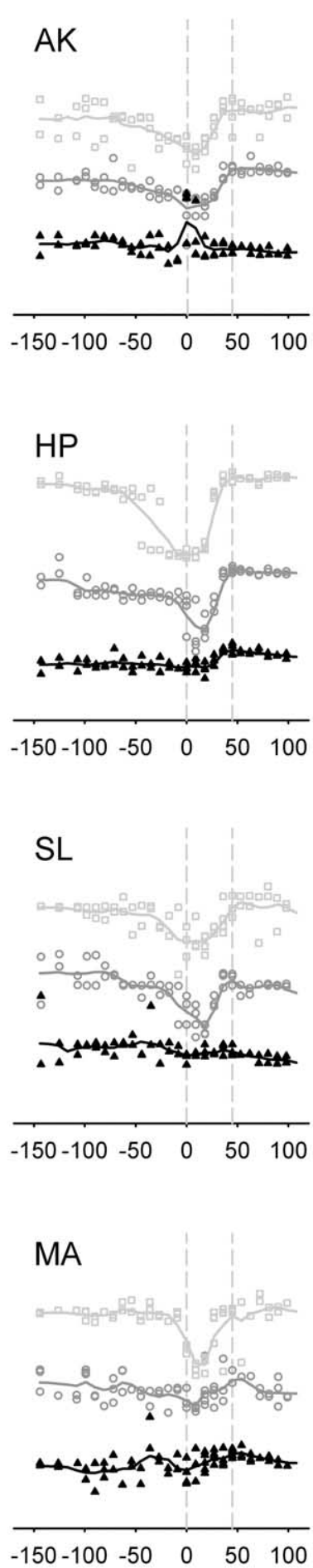

Time from saccade onset (ms)

Fig. 2. Individual responses of the four subjects in the real (left column) and simulated (right column) saccade task, aligned on motion onset. Bar localization is shown for bars presented at five (gray dots), -5 (dark gray dots) and $-15^{\circ}$ (black dots). Continuous curves are weighted running averages through the data with a time constant of $33 \mathrm{~ms}$.

simulated saccades in previous studies may, at least partially, have arisen from differences in retinal stimulation.
For instance, in the simulated saccade condition of the study of Honda (1995), the initial fixation point was extin- 

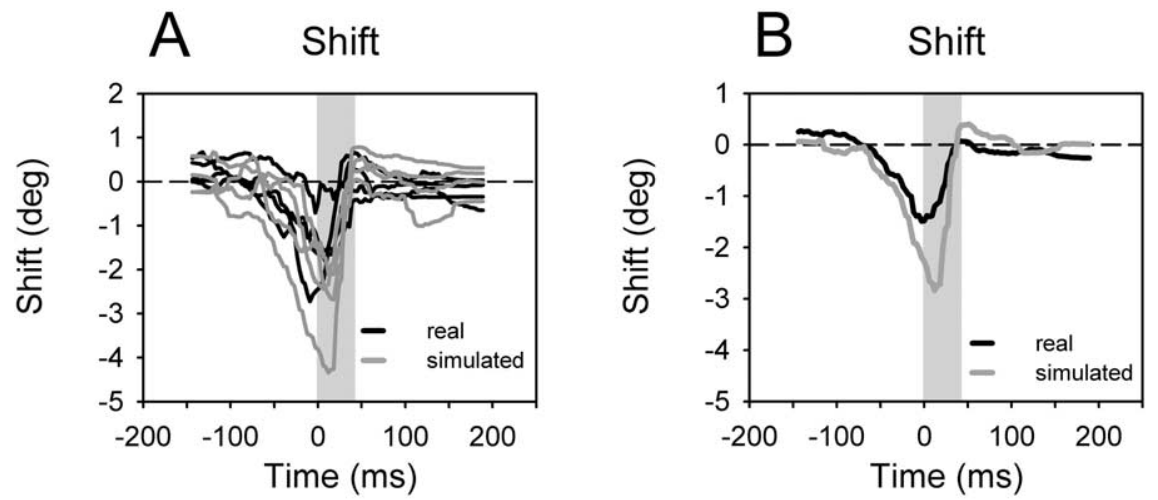

C Compression

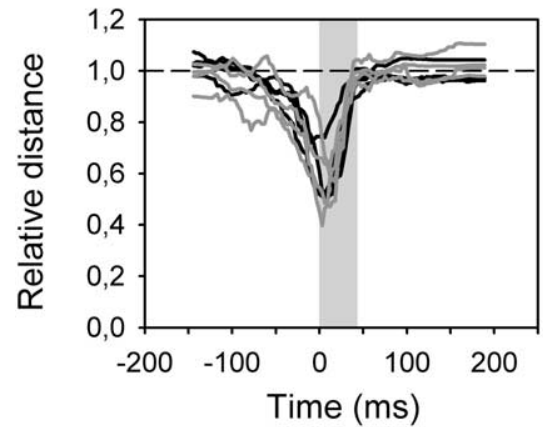

D Compression

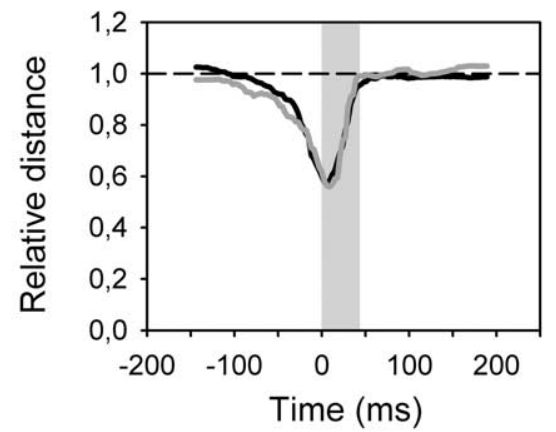

Fig. 3. Shift and compression of visual space around onset of real and simulated saccades. The shift index is defined as the mean of the three apparent positions of the bar relative to the mean $100 \mathrm{~ms}$ before and after image motion. The compression index is defined as the mean absolute separation between the three apparent bar position and saccade target relative to the mean absolute separation 100 ms before and after image motion. A value of 1 indicates no compression and a value of 0 maximal compression. Individual shift (A) and compression (B) values as a function of time and respective mean index curves $(C, D)$, averaged across subjects.

guished $200 \mathrm{~ms}$ before motion onset and no peripheral target was presented. To elucidate the factors leading to different patterns of mislocalization before saccade-like motion of the retinal image, we ran a series of further experiments, where we changed the availability of visual references around saccade-like image motion (Fig. 4A-D). Again we used multiple positions across the visual field and focused on the time of motion onset.

Fig. 5 shows results for the different conditions, averaged over subjects. Apparently, shift and compression are
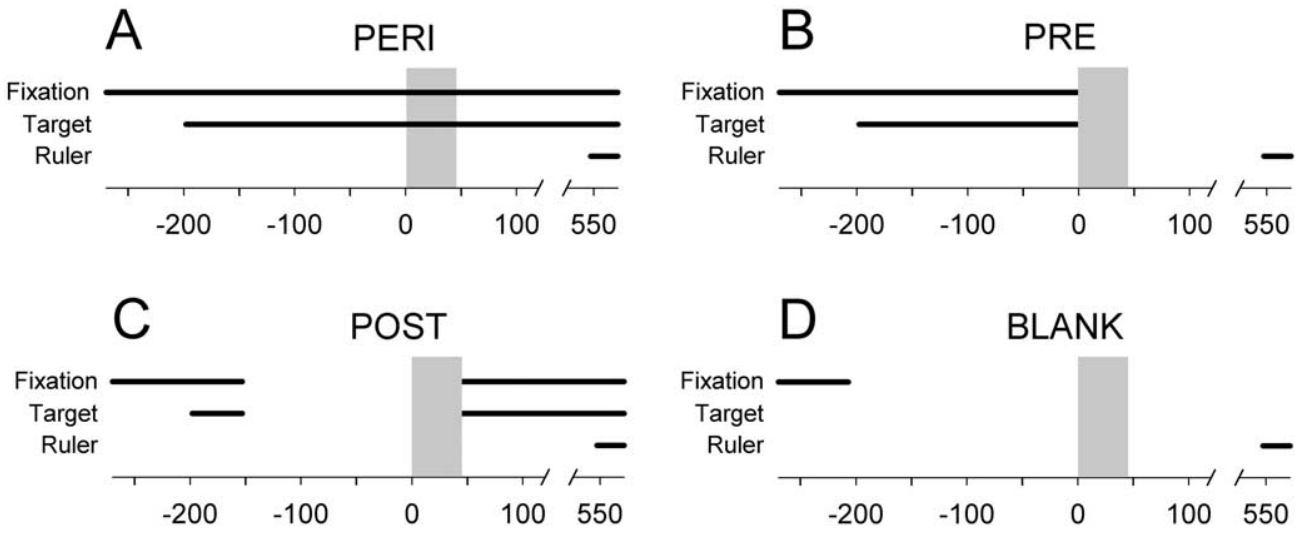

Fig. 4. Schematic of the four different simulated saccade conditions used to test the modulation of perceptual mislocalization by the availability of visual references. In the peri condition (A), references were available throughout the trial. In the pre (B) and post (C) condition, references were switched off at motion onset or not available from $150 \mathrm{~ms}$ before image motion until motion offset, respectively. In the blank condition (D), the initial fixation was switched off $200 \mathrm{~ms}$ before motion onset and no references besides the homogenous gray background area were available around image motion. 
A
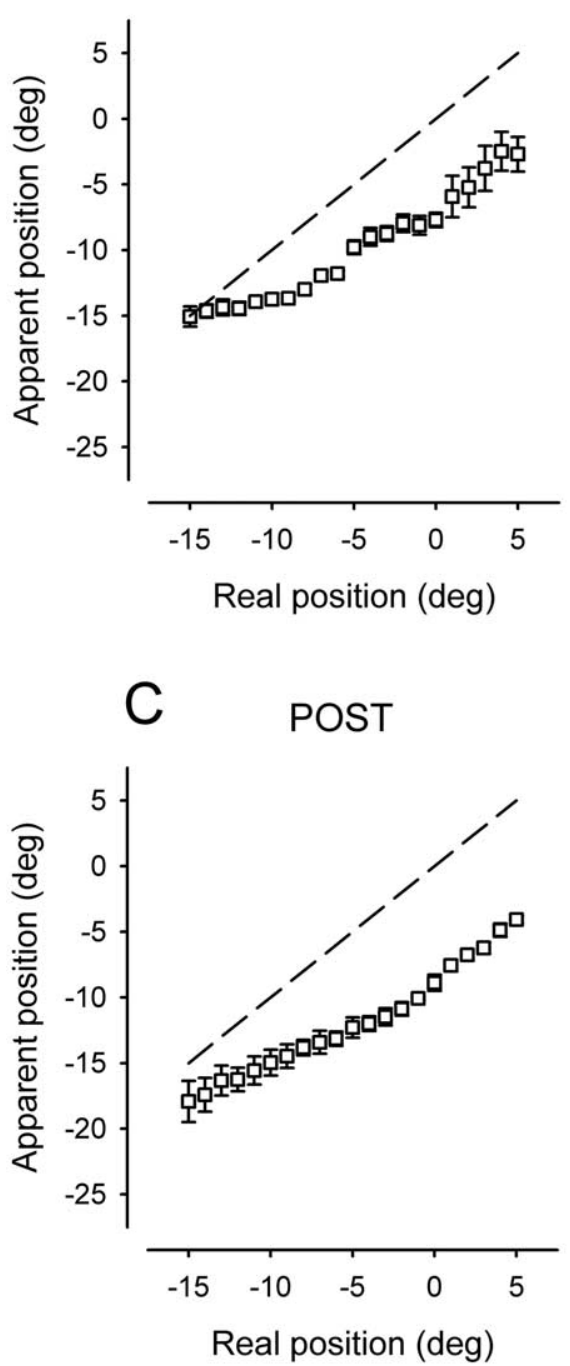

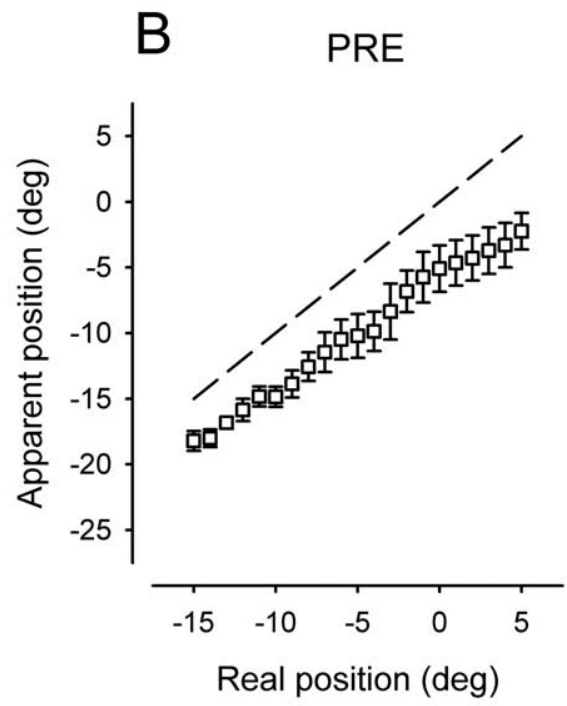

\section{BLANK}

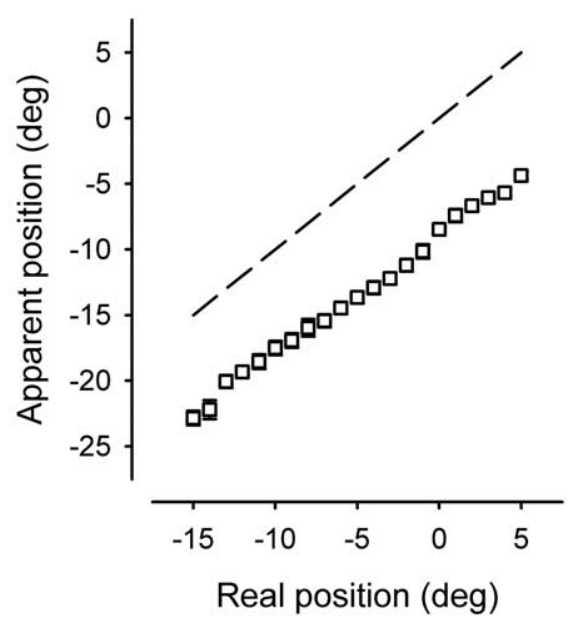

Fig. 5. Apparent positions of bars presented at multiple positions across the visual field just before saccade-like motion of the retinal image. We used 21 evenly spaced positions from $-15^{\circ}$ to $5^{\circ}$ eccentricity. The dashed line indicates veridical localization. Squares represent averaged median localization of four subjects ( \pm S.E.M). See Fig. 4A-D for explanation of the respective conditions.

differentially affected by the availability of visual references around image motion. For comparison, the original condition with initial fixation point and peripheral target presented throughout the trial is shown first (peri condition). Compared with this condition, compression is weaker with references selectively available before image motion (pre condition) and is virtually abolished when no further references besides the gray screen area are available around image motion (blank condition). By contrast, compression is comparable to the peri condition when references disappear $150 \mathrm{~ms}$ before image motion and reappear after image motion (post condition).

For easier comparison of differential effects we calculated shift and compression indices with the shift index calculated as described above (Fig. 6). Here, individual values were normalized with respect to veridical localization. Compression values were computed by regressing the apparent against real positions and estimating the slope (see also legend of Fig. 6). Apparently, the shift against image motion becomes larger with fewer references available before motion onset and approaches the amount of the retinal displacement in the blank condition (Fig. 6A). On the other hand, the presence of references after image motion mainly determines the amount of space compression (Fig. 6B). Differences in the respective shift and compression indices for the different conditions were significant (non-parametric Friedman-test, $P<0.02$ for shift indices, $P<0.01$ for compression indices, respectively).

\section{DISCUSSION}

In this study, we compared mislocalization patterns for stimuli briefly flashed before saccades and saccade-like motion of the retinal image. We found surprisingly similar 
A

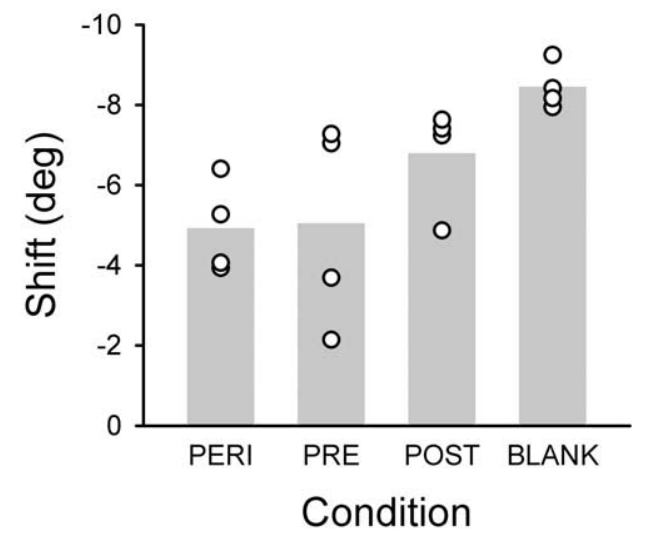

B Compression

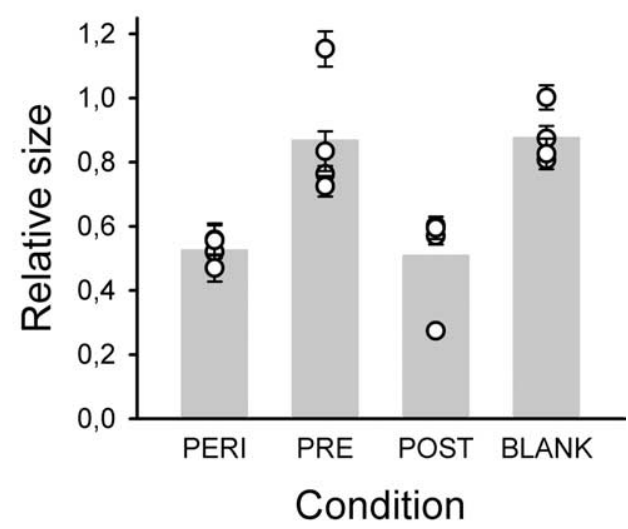

Fig. 6. Shift $(A)$ and compression (B) of visual space for different simulated-saccade conditions at motion onset. The shift index is defined as the mean of the apparent positions, normalized with respect to real position. Compression values were computed by regressing the apparent against real positions and estimating the slope, weighting each point by its standard error. The range of regression was limited to positions left of initial fixation. Thus, due to different analysis and normalization, absolute values cannot directly be compared with data obtained in the first experiment (see Fig. 3 ). Bars show averaged index values from the four subjects, symbols show individual index values (with error bars in Fig. 6B indicating the standard error of the slope estimation).

spatio-temporal dynamics of mislocalization in both cases. This stands in contrast to previous studies where clearly different patterns of mislocalization before real and simulated saccades were observed. In these studies, mislocalization before saccade-like motion of the retinal image was found as a uniform shift against image motion (Honda, 1995; Morrone et al., 1997), that tended to start earlier and be of greater magnitude than before real saccades (Morrone et al., 1997). In particular, no compression of space was found for simulated saccades (Honda, 1995; Morrone et al., 1997).

Our findings from simulated saccades indicate that the availability of visual references around image motion strongly influences the specific pattern of mislocalization. Consistent with mislocalization patterns before real saccades (Lappe et al., 2000), the presence of visual references after saccade-like image motion appears to determine the amount of space compression before motion onset. When no visual references are available at all around image motion, the pattern of mislocalization before image motion resembles the findings of Honda (1995) of a strong and more uniform shift of apparent position against image motion for simulated saccades. Thus, we hypothesize that different patterns of mislocalization for real and simulated saccades may, at least partially, have arisen from differences in retinal stimulation (Honda, 1995). Differences in comparison to the results of Morrone and coworkers (1997) remain more difficult to explain. Our results suggest that the presence of a peripheral target in a critical time before and especially after the retinal image motion plays an important role in the emergence of space compression with simulated saccades. Note however, that in comparison to the findings of Morrone et al. (1997) with real saccades, the localization bias toward the peripheral target is less pronounced for simulated saccades in our study. Since space compression also tended to be weaker in our real saccade task, different stimulus parameters, such as luminance (Awater and Lappe, 2004), contrast level (Michels and Lappe, 2004) and spatial frequency of the flashed stimulus may primarily account for this difference. Further experiments probing mislocalization shortly before real and simulated saccades with a parametric modulation of these factors are required to resolve the difference. In this context, it remains an open question whether our findings generalize to conditions that encourage localization independent from allocentric references, such as in a vernier task in which the distance between two successively flashed bars has to be reported (Morrone et al., 1997).

Differences in the pattern of mislocalization before real and simulated saccades also emerged in our study, where the maximum shift was larger in the simulated saccade task. These differences are consistent with the findings of Morrone and coworkers (1997) and could indicate the presence of different factors contributing to localization in both tasks (e.g. changes in an extraretinal eye position signal before real but not before simulated saccades). Alternatively, since the temporal dynamics of perceptual mislocalization were almost identical, the same factors may be involved in both tasks, albeit to a different degree. For instance, differences in retinal stimulation (e.g. smooth vs. apparent motion of the retinal image during real and simulated saccades), differences in the covert orienting of attention or saccade planning toward the peripheral target (Deubel and Schneider, 1996) may likely lead to differences in perceptual mislocalization in both tasks. A further analysis of these factors on perceptual mislocalization was beyond the scope of our study.

Our findings cast doubt on a decisive contribution of an extraretinal eye position signal to perisaccadic mislocalization in our experiment. Although extraretinal signals may modulate the particular dynamics of perisaccadic mislocal- 
ization, we propose that visual factors may mainly account for the basic pattern of mislocalization, at least under conditions of normal illumination. A possibly underestimated explanation for mislocalization may lie in the way briefly flashed stimuli are processed by the visual system (Schlag and Schlag-Rey, 2002): The common attribution of perisaccadic mislocalization to an extraretinal signal presupposes a faithful neural representation of a briefly flashed stimulus, both in the spatial and temporal domain. However, the buildup of visual representations is subject to variable afferent delays (Schmolesky et al., 1998). More decisive in the context of perisaccadic mislocalization may be the phenomenon of visible persistence. Psychophysical (Bowen et al., 1974; Coltheart, 1980) and electrophysiological (Duysens et al., 1985; Kratz and May, 1990) studies indicate that the visual percept and corresponding burst of neuronal activity following a briefly flashed stimulus may last for up to multiples of its presentation time. Visible persistence could explain a presaccadic shift of apparent positions in saccade direction: Persistence of the retinotopic stimulus trace may lead to erroneous integration of a presaccadically flashed stimulus position within an already moving or postsaccadic visual reference frame (Schlag and Schlag-Rey, 2002; Pola, 2004). In this view, a perisaccadic shift of perceived positions could in principle relate to the so-called flash-lag effect. It refers to the observation that a flashed stimulus is commonly reported to lag a physically aligned moving stimulus (Nijhawan, 2002; Schlag and Schlag-Rey, 2002).

What additional process may explain a compression of visual space? Retinal image motion is known to induce a suppression of visible persistence, presumably due to lateral inhibitory interactions in retinotopically organized visual areas (Burr, 1980; Hogben and DiLollo, 1985). Onand offset of visual references around image motion may further act to shorten the visible persistence of the flashed bar. This would in turn lead to a reduced shift of apparent direction of the flashed bar. Indeed, the shift of apparent positions strongly depends on the availability of visual references before image motion (Fig. 6A). Masking a stimulus by visual motion signals and thereby shortening the time over which it can be integrated will furthermore result in a weaker representation of its position. A less reliable representation of the flashed bar position may lead to an additional localization bias toward salient references. This would explain why the availability of visual references especially after image motion exerts a powerful localization bias of the flashed bar toward these references and thus mainly determines the magnitude of space compression (Fig. 6B). Support for a dependency of space compression on stimulus strength comes from a recent study: Michels and Lappe (2004) showed the localization bias toward the saccade target to depend on stimulus contrast: Smaller contrast of the flashed bar resulted in an increased compression of visual space. Furthermore, the time-course and strength of space compression correlated with the detection rate of the flashed stimulus. This indicates that presaccadic space compression may result from an al- ready faint stimulus representation that is further weakened around saccade onset (Michels and Lappe, 2004).

Single-neuron recordings in medial temporal and medial superior temporal areas of awake monkeys support the hypothesis of a visual origin of perisaccadic mislocalization (Krekelberg et al., 2003). The perisaccadic position encoding of a flashed stimulus was found to be disturbed in a manner consistent with a compression of visual space toward the saccade target. Most critically to our results, mislocalization was found in a retinal frame of reference, indicating that encoding in retinotopic coordinates is already inaccurate around saccade onset. The authors state that "a nonlinear interaction of the saccade-induced visual stimulation (by the background, the saccade target, or other visual references) with the stimulation by a flashed bar" (Krekelberg et al., 2003; p. 543) may be one explanation for mislocalization. This explanation would comply with our hypothesis on a predominantly visual origin of perisaccadic mislocalization.

While extraretinal eye position signals may ensure accurate space perception in the dark (Hansen and Skavenski, 1977; Matin et al., 1982; Burr et al., 2001), space constancy under normal illumination may be accomplished by a comparison of the pre- and postsaccadic visual reference frame (MacKay, 1970; O'Regan, 1984; Deubel et al., 1996; McConkie and Currie, 1996). This seems ecologically advantageous, since behaviorally relevant stimuli are typically available for longer periods of time. Localization of stable stimuli relative to salient references is presumably more accurate than the reliance on an extraretinal signal which, by virtue of its low gain, may not fully compensate for retinal displacements (Bridgeman, 1995). In this view, the typical experimental setup for assessing perisaccadic space constancy reveals a flaw in the predominating mechanism of relative localization: Briefly flashed stimuli are grossly mislocalized before fast motion of the retinal image.

Acknowledgments-Supported by the Deutsche Forschungsgemeinschaft (grant PI 248/2-1).

\section{REFERENCES}

Awater H, Lappe M (2004) Perception of visual space at the time of pro- and antisaccades. J Neurophysiol 91:2457-2464.

Bowen RW, Pola J, Matin L (1974) Visual persistence: Effects of flash luminance, duration and energy. Vision Res 14:295-303.

Bridgeman B (1995) A review of the role of efference copy in sensory and oculomotor control systems. Ann Biomed Eng 23:409-422.

Burr D (1980) Motion smear. Nature 284:164-165.

Burr D, Morrone MC, Ross J (2001) Separate visual representations for perception and action revealed by saccadic eye movements. Curr Biol 11:798-802.

Cai RH, Pouget A, Schlag-Rey M, Schlag J (1997) Perceived geometrical relationships affected by eye-movement signals. Nature 386 : 601-604.

Coltheart M (1980) Iconic memory and visible persistence. Percept Psychophys 27:183-228.

Deubel H, Schneider WX (1996) Saccade target selection and object recognition: evidence for a common attentional mechanism. Vision Res 36:1827-1837.

Deubel H, Schneider WX, Bridgeman B (1996) Postsaccadic target blanking prevents saccadic suppression of image displacement. Vision Res 36:985-996. 
Duysens J, Orban GA, Cremieux J, Maes H (1985) Visual cortical correlates of visible persistence. Vision Res 25:171-178.

Hansen RM, Skavenski AA (1977) Accuracy of eye position information for motor control. Vision Res 17:919-926.

Hogben JH, DiLollo V (1985) Suppression of visible persistence in apparent motion. Percept Psychophys 38:450-460.

Honda H (1989) Perceptual localization of visual stimuli flashed during saccades. Percept Psychophys 45:162-174.

Honda H (1993) Saccade-contingent displacement of the apparent position of visual stimuli flashed on a dimly illuminated structured background. Vision Res 33:709-716.

Honda H (1995) Visual mislocalization produced by a rapid image displacement on the retina: examination by means of dichotic presentation of a target and its background scene. Vision Res 35:3021-3028.

Horwitz GD, Albright TD (2003) Short-latency fixational saccades induced by luminance increments. J Neurophysiol 90:1333-1339.

Kratz KE, May JG (1990) Response persistence of cat retinal ganglion cells to the temporally discrete presentation of sinewave gratings. Int J Neurosci 52:111-119.

Krekelberg B, Kubischik M, Hoffmann K-P, Bremmer F (2003) Neural correlates of visual localization and perisaccadic mislocalization. Neuron 37:537-545.

Lappe M, Awater H, Krekelberg B (2000) Postsaccadic visual references generate presaccadic compression of space. Nature 403: 892-895.

MacKay DM (1970) Mislocation of test stimuli during saccadic image displacement. Nature 227:731-733.

Martinez-Conde S, Macknik SL, Hubel DH (2004) The role of fixational eye movements in visual perception. Nat Rev Neurosci 95: 229-240.

Matin L, Pearce DG (1965) Visual perception of direction for stimuli during voluntary saccadic eye movements. Science 148:14851488.

Matin L, Picoult E, Stevens JK, Edwards MW, Young D, MacArthur R (1982) Oculoparalytic illusion: visual-field dependent spatial mislocalizations by humans partially paralyzed with curare. Science 216:198-201.
McConkie G, Currie CB (1996) Visual stability across saccades while viewing complex pictures. J Exp Psychol Hum Percept Perform 22:563-581.

Michels L, Lappe M (2004) Contrast dependency of saccadic compression and suppression. Vision Res 44:2327-2336.

Morrone MC, Ross J, Burr D (1997) Apparent position of visual targets during real and simulated saccadic eye movements. J Neurosci 17:7941-7953.

Nijhawan R (2002) Neural delays, visual motion and the flash-lag effect. Trends Cogn Sci 6:387-393.

O'Regan JK (1984) Retinal versus extraretinal influences in flash localization during saccadic eye movements in the presence of a visible background. Percept Psychophys 36:1-14.

Pola J (2004) Models of the mechanism underlying perceived location of a perisaccadic flash. Vision Res 44:2799-2813.

Reppas JB, Usrey WM, Reid RC (2002) Saccadic eye movements modulate visual responses in the lateral geniculate nucleus. Neuron 29:961-974.

Ross J, Morrone MC, Burr D (1997) Compression of visual space before saccades. Nature 386:598-601.

Ross J, Morrone MC, Goldberg ME, Burr D (2001) Changes in visual perception at the time of saccades. Trends Neurosci 24:113-121.

Schlag J, Schlag-Rey M (2002) Through the eye, slowly: delays and localization errors in the visual system. Nat Rev Neurosci 3:191-200.

Schmolesky MT, Wang Y, Hanes DP, Thompson KG, Leutgeb S, Schall JD, Leventhal AG (1998) Signal timing across the macaque visual system. J Neurophysiol 79:3272-3278.

Sperling G (1990) Comparison of perception in the moving and stationary eye. In: Eye movements and their role in visual and cognitive processes: Reviews of oculomotor research, Vol. 4 (Kowler E, ed), pp 307-351. Amsterdam: Elsevier.

Sperry RW (1950) Neural basis of the spontaneous optokinetic response produced by visual inversion. J Comp Physiol Psychol 43:482-489.

von Helmholtz H (1866) Handbuch der Physiologischen Optik. Leipzig: Voss.

von Holst E, Mittelstaedt H (1950) Das Reafferenzprinzip (Wechselwirkung zwischen Zentralnervensystem und Peripherie). Naturwissenschaften 37:464-476. 